\title{
THE MODELLING OF LARGE STRAIN TEXTURES AND STRESS-STRAIN RELATIONS OF POLYETHYLENE
}

\author{
A. DAHOUN ${ }^{+}$, G.R. CANOVA*, A. MOLINARI*, \\ M.J. PHILIPPE**, Ch. G'SELL ${ }^{+}$ \\ + Ecole des Mines, Parc de Saurupt, 54042 Nancy Cedex \\ * LPMM, ** LM2P, Univ de Metz, Ile du Saulcy, 57045 Metz Cedex 1
}

\section{1 - INTRODUCTION}

The experimental stress-strain curves of a polyethylene in uniaxial tension exhibits an increase of the hardening with the strain, while in simple shear a slight softening is observed.

It is the scope ot this paper to : 1) identify the micro mechanisms at the origin of the observed evolution of hardening, 2) propose a micromacro modelling which describes the evolution of the internal structure of the material and the overall stress-strain relations.

\section{2 - MATERIAL}

A high density polyethylene obtained by extrusion is considered. Two phases are present : an amorphous phase and a crystalline phase. The crystalline part has a volume fraction of $71 \%$ and an orthorhombic crystallographic structure with $\mathrm{a}=7,4 \mathrm{~A}^{\circ}, \mathrm{b}=4,94 \mathrm{~A}^{\circ}$ and $\mathrm{c}=2,54 \mathrm{~A}^{\circ}$ as lattice parameters.

This crystalline part is constituted by platelets with a strong aspect ratio ( width $=$ length $=500 \mathrm{~A}^{\circ}$, thickness $=25 \mathrm{~A}^{\circ}$ ), each platelet beeing a collection of macromolecular chains (fig. 1). The platelets are gathered into clusters, named spherolites, having a radius of the order of $1 \mu \mathrm{m}$. In the initial state, a platelet is orthogonal to a radial direction of a spherolite, and the platelets are equally distributed in all directions (fig. 2), so that the initial behavior of a spherolite is nearly isotropic. The interval between the platelets is filled with the amorphous phase. 
Chain slips are the elementary mechanisms of plastic deformation of a platelet (fig. 3). Since there are not enough mechanisms to accomodate any deformation, the flow surface of a platelet is not closed.

\section{3 - MECHANICAL TESTS}

The results of the modelling will be compared with two mechanical tests (tension and simple shear) performed at room temperature at a constant equivalent strain rate $\frac{\dot{\varepsilon}}{\varepsilon}=5 \times 10^{-4} \mathrm{~s}^{-1}$ where $\dot{\varepsilon}=\left(2 / 3 \dot{\varepsilon}_{\mathrm{ij}} \dot{\varepsilon}_{\mathrm{ij}}\right)^{1 / 2}$. Details on this tests can be found in Dahoun and G'sell [1]. On fig. 4, stressstrain curves are presented in term of Mises work conjugated quantities for the tension test, $\sigma$ vs $\varepsilon=\ln 1 / 10$; and for the simple shear, $\tau \sqrt{3}$ vs $\gamma / \sqrt{3}$; where $\tau$ and $\gamma$ are the shear stress and the shear strain. It is observed that for moderate deformations $(\leq 0.4)$ the stress strain curves are almost identical. For this range of deformations the material seems therefore to present a Mises isotropic behavior. However for larger deformations a strong deviation in the responses appears, since the tension test exhibits an important hardening, while a little softening appears in the shear test. The following question now arises : what is the evolution of the internal structure responsible of the development of such different behaviors?

\section{4 - MODELLING}

An induced anisotropy is developing for large deformations. The strong anisotropy in the mechanical behavior of the platelets, due to the orthorhombic crystallographic texture, is transfered at the macroscopic level via :

- the crystallographic texture

- the morphological texture (orientation of the platelets).

In the initial state, the orientation of the crystallographic lattices and the orientation of the geometrical axes of the platelets are isotropic. This results in an isotropic overall behavior of the material. The deformation induces an evolution of the crystallographic texture as well as an evolution of the morphological texture (reorientation of the platelets).

A modelling of the development of the internal structure and of the induced overall anisotropy is now proposed. In this modelling the following simplifications are made :

- the effect of the amorphous phase is neglected

- elasticity is neglected

- the elementary mechanisms of plastic deformation (chain slip) exhibits no hardening

- the shear rate $i s$ associated to the slip system $s$ is related to the resolved stress $\tau^{(s)}$ by a nonlinear viscous law. 
(1)

$$
\frac{\tau^{(\mathrm{s})}}{\tau^{\mathrm{o}}}=\left(\frac{\dot{\gamma}^{(\mathrm{s})}}{\dot{\gamma}^{\mathrm{o}}}\right)^{\mathrm{m}}
$$

where $\tau^{0}$ and $\gamma^{0}$ are some reference stress and strain rate characteristic of the material.

It is assumed that the overall velocity gradient $L$ is given $\left(L_{i j}=\partial v_{i} / \partial x_{j}\right.$ where $y$ is the velocity of a particle). A 1-site viscoplastic self consistent scheme is used to calculate the evolution of the internal structure and to determine the effective properties of the material, see A. Molinari et al [2] and Ahzi et al [3] for details. For each platelet g, considered as embedded in the homogeneous equivalent medium having the effective properties of the crystalline part of the polyethylene, an interaction law is obtained:

$$
\underline{S}^{\mathrm{g}}-\underline{\overline{\mathrm{S}}}=\left(\Gamma_{\mathrm{gg}}^{-1}+\underline{\mathrm{A}}^{0}\right)\left(\underline{\mathrm{D}}^{\mathrm{g}}-\underline{\overline{\mathrm{D}}}\right)
$$

where $\underline{\bar{D}}=1 / 2(\underline{L}+\underline{L})$ is the overall applied strain rate, and $\overline{\mathrm{S}}$ is the overall deviatoric Cauchy stress $; \underline{D} g$ is the strain rate (assumed uniform) and $\underline{S} \mathbf{g}$ is the deviatoric stress in the platelet $g ; \underline{A}^{\circ}$ is the effective tangent modulus, and $\Gamma^{\mathrm{gg}}$ is an interaction tensor taking account of the effective tangent modulus $\mathrm{A}^{\circ}$ and of the shape of the platelet. In the simplified version used here, one takes for the homogeneous equivalent medium an isotropic tangent modulus :

$$
\underline{A}^{\circ}=\mu \underline{I}
$$

where $I$ is a fourth order identity tensor, and $\mu$ is an unknown effective property. It should be noted that $\mu$ depends on the applied strain rate $\overline{\mathrm{D}}$. Using (3), the interaction law (2) reads :

$$
\underline{S}^{\mathrm{g}}-\underline{\bar{S}}=\mu \underline{K}^{\mathrm{g}}\left(\underline{\bar{D}}-\overline{\mathrm{D}}^{\mathrm{g}}\right)
$$

where the tensor:

$$
\underline{\mathrm{K}}^{\mathrm{g}}=\left(\frac{1}{\mu} \Gamma_{\mathrm{gg}}^{-1}+\underline{\mathrm{I}}\right)
$$

only depends on the morphology of the platelet. The constitutive law of a platelet reads :

$$
\begin{aligned}
\underline{\mathrm{D}}^{\mathrm{g}} & =\underline{\mathrm{F}}\left(\mathrm{S}^{\mathrm{g}}\right) \\
& =\Sigma{ }_{\mathrm{s}} \frac{1}{2}\left(\underline{s}^{(\mathrm{s})} \otimes \underline{\mathrm{m}}^{\mathrm{s}}+\underline{\mathrm{m}}^{(\mathrm{s})} \otimes \underline{s}^{(s)}\right) \dot{\gamma}_{0}\left(\frac{\tau^{(\mathrm{s})}}{\tau^{0}}\right)^{1 / \mathrm{m}}
\end{aligned}
$$

where $\mathrm{s}^{(\mathrm{s})}$ and $\mathrm{m}^{(\mathrm{s})}$ are the slip direction and the normal to the slip plane. Assuming that $\overline{\mathrm{D}}$ and $\underline{K}^{\mathrm{g}}$ are known, the strain rate and the deviatoric 
stress can be calculated in the platelet $g$ in term of $\underline{\bar{S}}$ and $\mu$ using (4) and (6) :

$$
\left\{\begin{array}{l}
\underline{S}^{\mathrm{g}}=\underline{G}(\overline{\mathbf{S}}, \mu) \\
\underline{D}^{\mathrm{g}}=\underline{\mathrm{F}}\left(\underline{\mathbf{S}}^{\mathrm{g}}\right)=\underline{\mathrm{F}}(\underline{\mathrm{G}}(\overline{\mathbf{S}}, \mu))
\end{array}\right.
$$

Then $\mu$ and $\underline{S}$ are calculated in order to satisfy the following self consistency conditions :

$$
\begin{aligned}
& <\underline{\mathrm{D}}^{\mathrm{g}}>=\overline{\mathrm{D}} \\
& \left\langle\underline{\mathrm{S}}^{\mathrm{g}}: \mathrm{D}^{\mathrm{g}}\right\rangle=\overline{\mathrm{S}}: \overline{\mathrm{D}}
\end{aligned}
$$

where $<$. $>$ represents the volumic average.

Remark: If the inclusions had homothetic shapes and parallel principal axes, $K^{g}$ would be independent of $g$. From (4) and (8) the following result would follow:

$$
\left\langle\underline{\mathbf{S}}^{\mathrm{g}}\right\rangle=\overline{\mathbf{S}}
$$

Here, since the afore mentioned conditions on the platelets are not satisfied, the validity of the results must be ensured by a direct check of the condition (9) during the calculation.

From $\underline{S}$, the shear rates $\gamma^{(s)}$ can be calculated with (1) and then the crystallographic texture and the orientations of the platelets can be updated, see [2] [3].

\section{5 - RESULTS}

The simulated stress-strain curves represented on fig. 5 are in good agreement with the experimental curves shown on fig. 4 . It can be noted that the elastic effects are only appearing at the begining of the deformation process.

Moreover the evolution of the anisotropic hardening can be explained from the changes in the internal structure.

It is shown here how the evolutions of the crystallographic texture and of the morphological texture both contribute to the development of the material anisotropy. This is illustrated for the uniaxial tension test. The discussion for the shear test will be presented elsewhere.

The fig. 6 is a (022) pole figure, at the uniaxial deformation $\varepsilon=1$ obtained by numerical simulations. It is shown that the normal to the (011) plane makes an angle comprised between $25^{\circ}$ and $30^{\circ}$ with the tensile direc- 
tion. The irregularity of the lines is due to the fact that the size of the crystallites are large in the sample analysed here. On the other hand it is known that the $c$ axis of the chains makes an angle of about $27^{\circ}$ with the normal to the (011) plane. It is noted furthermore, as shown by the (200) simulated pole fig. 7, that the tensile axis is parallel to the (200) planes. Thus the direction $\mathrm{c}$ must be perpendicular to the (200) direction and have an angle of $27^{\circ}$ with the (011) direction. Therefore, it can be concluded that the chain axes $\mathrm{c}$ tend to become parallel to the tensile axis. Since the chain axis $\mathrm{c}$ is the hard direction in a platelet, the evolution of these axes towards the tensile direction renders the platelets more difficult to deform. This is the contribution of the crystallographic texture evolution to the development of the strong hardening in the tensile test. The experimental (022) and (200) pole figures, fig. 8, compared to fig. 6 and 7 confirm the validity of the results of the modelling.

Another contribution to the hardening, is due to the rotation of the principal geometrical axes of the platelets. The numerical calculations show that the platelets tend to become parallel to the tensile axis. This results in a strengthening effect (like in composite materials). Therefore the evolution of the morphological texture leads to an additional hardening.

1. Dahoun, C. G'sell, to appear.

2. A. Molinari, G.R. Canova and S. Ahzi, Acta Metall., 35, 12 (1987).

3. S. Ahzi, A. Molinari and G.R. Canova, Yielding, Damage, and Failure of Anisotropic Solids, EGF5 (Ed. by J.P. Boehler), (1990), Mech. Eng. Publ., London, p. 425-441.
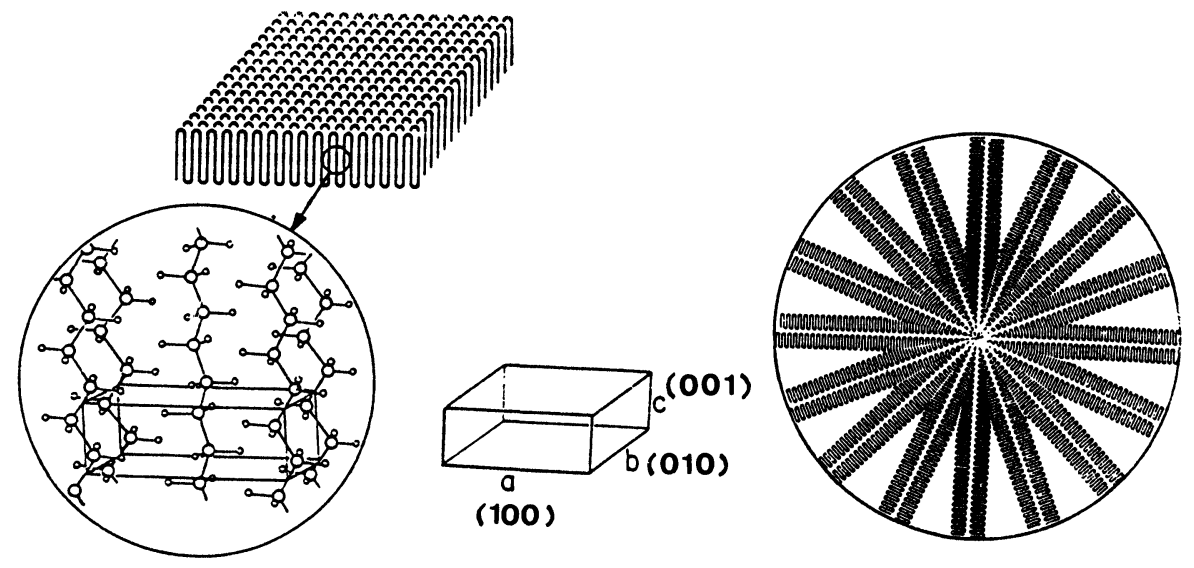

Figure 1: Structure of the HDPE crystallite. The crystal symmetry is orthorhombic, the $\mathrm{c}$ axis being aligned with the chain axes. 

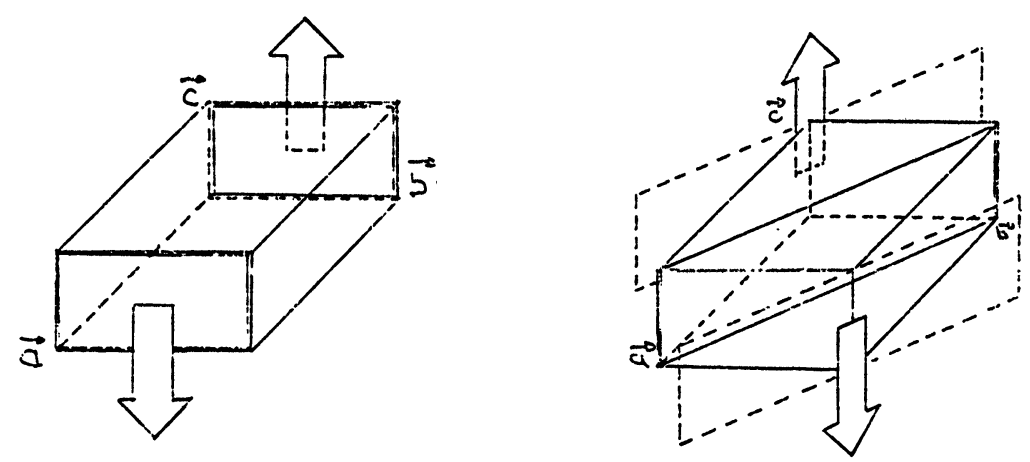

Figure 3: Schematic representation of two kinds of chain slips.

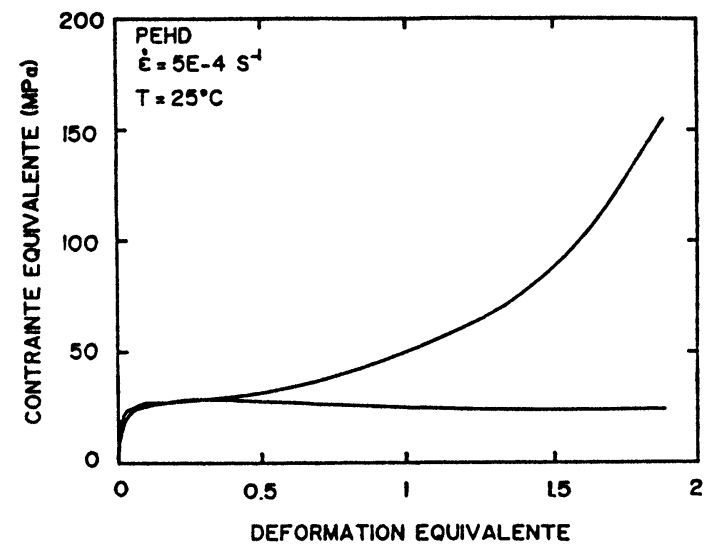

Figure 4: Mechanical stress/strain response of a HDPE material in 1 xial tension and simple shear. Note the increasing discrepancy.

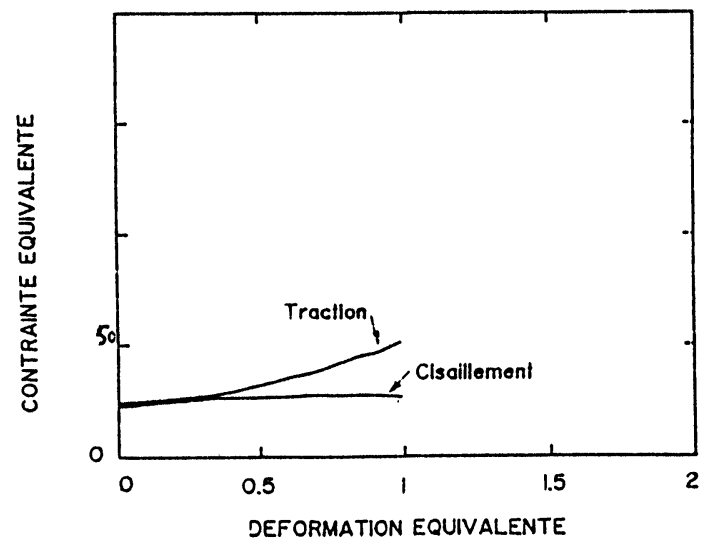

Figure 5: Stress/strain curves in uniaxial tension and simple shear obtained by the VPSC model up to a von Mises strain of 1 . Beyond that strain a numerical divergence is obtained in uniaxial tension. 


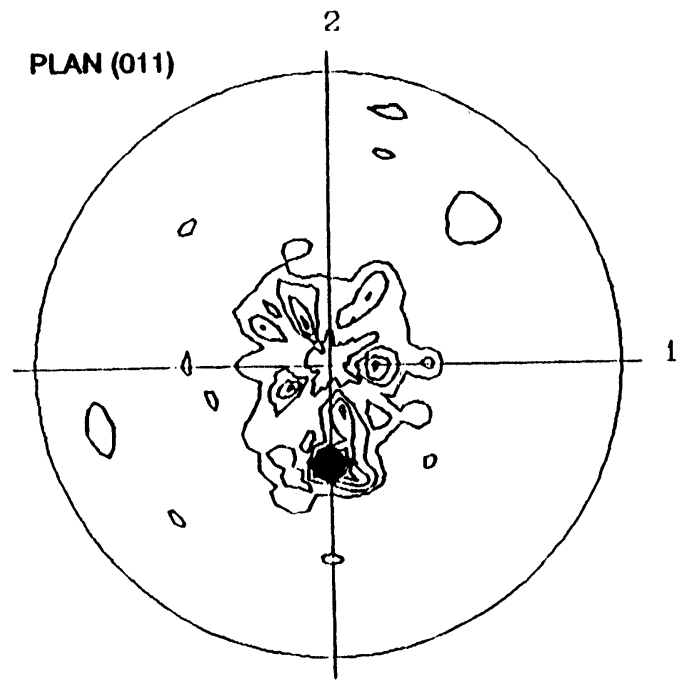

Figure 6: (022) pole figure obtained by the VPSC model in uniaxial tension at a strain of 1 .

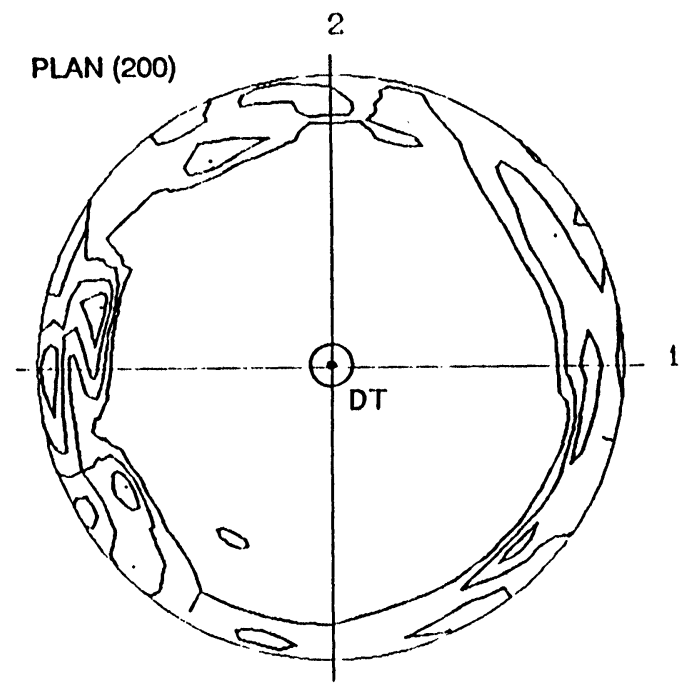

Figure 7: (200) pole figure obtained by the VPSC model in uniaxial ten. sion at a strain of 1 . 


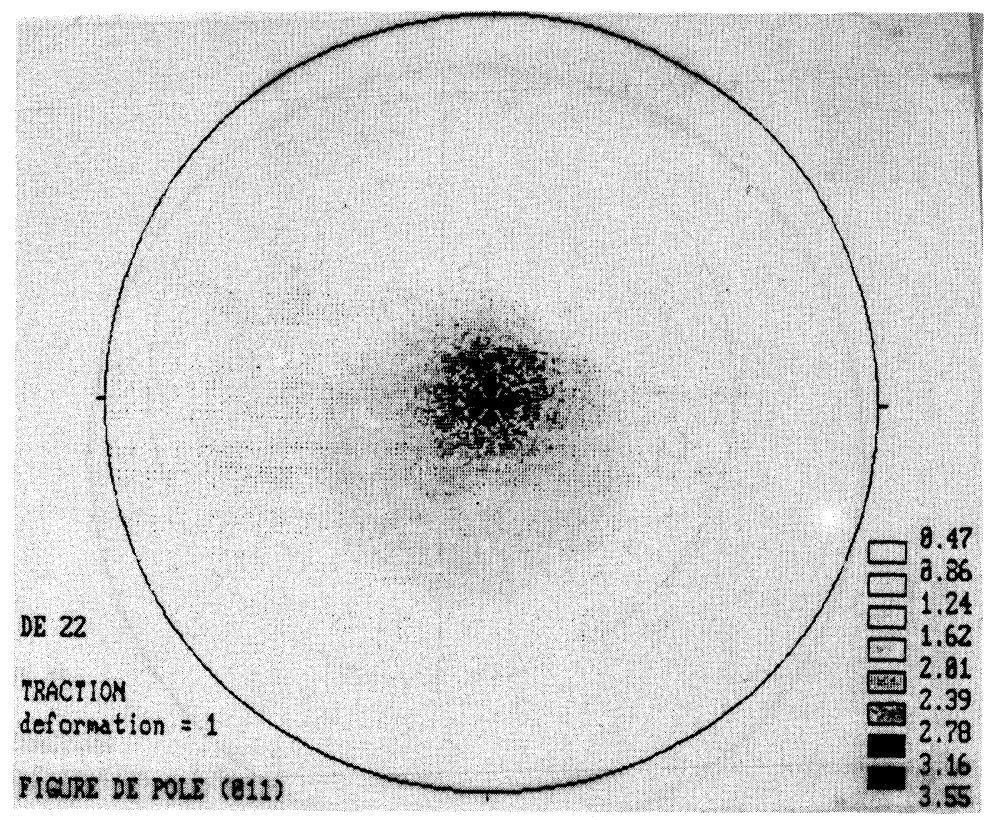

Figure 8a: (022) pole figure obtained experimentally in HDPE drawn at a strain of 1 .

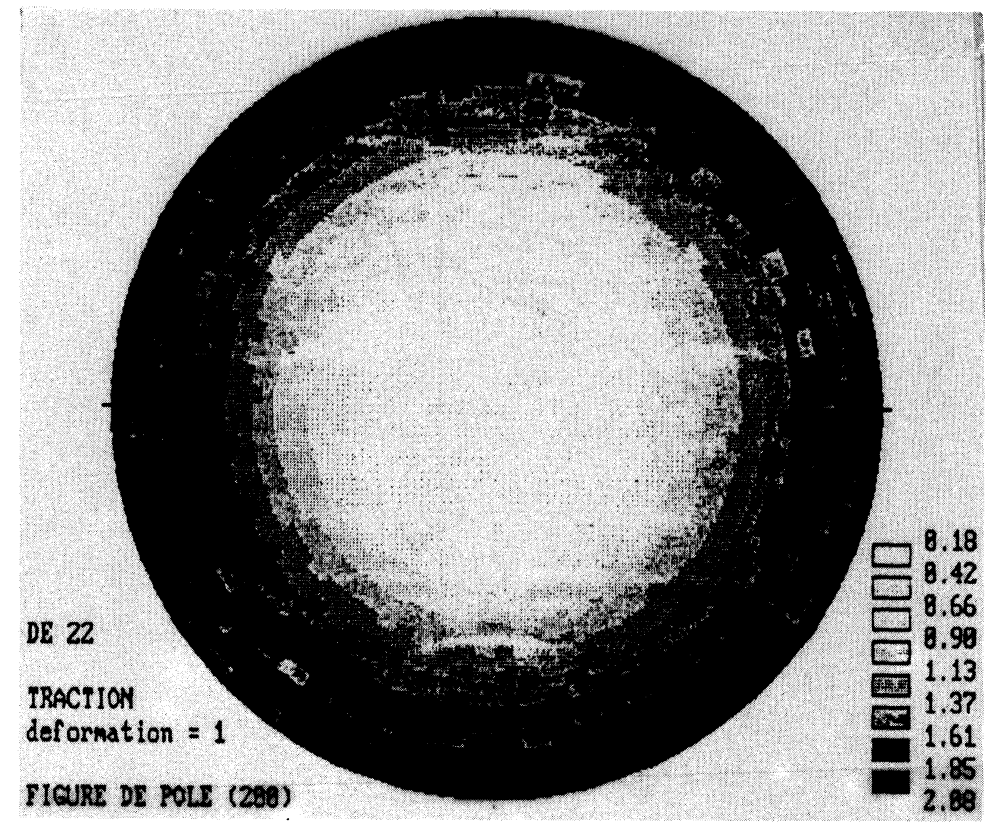

Figure 8b: (200) pole figure obtained experimentally in HDPE drawn at a strain of 1 . 